\title{
Percepción de la comunidad universitaria sobre el consumo de sustancias psicoactivas en la Universidad de Antioquia, Medellin, Colombia
}

\author{
Perception of University Community of Psychoactive Substance Abuse in the University \\ of Antioquia, Medellin, Colombia \\ Percepção da comunidade universitária sobre o consumo de sustâncias psicoativas na Universidade de \\ Antioquia, Medellín, Colômbia \\ Isabel Cristina Posada Psic MSc, Eneida Puerta-Hennao Psic, ${ }^{2}$ Eliana María Alzate Psic, ${ }^{2}$ Paola Andrea Oquendo Psic ${ }^{2}$ \\ Recibido: 8 de julio de $2013 \bullet$ Aceptado: 20 de junio de 2014 \\ Doi: dx.doi.org/10.12804/revsalud12.03.2014.09 \\ Para citar este artículo: Posada IC, Puerta-Henao E, Alzate EM, Oquendo PA. Percepción de la comunidad universitaria sobre el consumo de sustancias psicoactivas en la \\ Universidad de Antioquia, Medellín, Colombia Rev Cienc Salud. 2014;12(3):411-22. doi: dx.doi.org/10.12804/revsalud12.03.2014.09
}

\begin{abstract}
Resumen
Objetivos: Analizar la percepción de la comunidad universitaria sobre el contexto y los factores de riesgo asociados al consumo de sustancias psicoactivas. Materiales y métodos: Enfoque histórico hermenéutico, diseño cualitativo, teoría fundamentada como método de investigación. Para la recolección se usó la entrevista semiestructurada. El muestreo fue teórico. Se realizaron 18 entrevistas a la comunidad universitaria y a los profesionales de Bienestar Universitario. Para el análisis se utilizó la codificación, categorización e interpretación. Resultados: Se reconoce a la universidad como una institución de calidad en educación e investigación donde se asume una posición ante la vida, la ciudad y la academia y, a su vez, como un escenario con elementos que facilitan el consumo: espacio físico, imaginarios, estilos de vida, lo que simboliza ser universitario y el expendio de drogas. Conclusión: El consumo es, por lo tanto, un fenómeno que debe ser abordado desde una perspectiva que trascienda de lo local a lo nacional e internacional.
\end{abstract}

Palabras clave: Sustancias psicoactivas, factores de riesgo, estudiantes universitarios.

\footnotetext{
1 Facultad Nacional de Salud Pública, Universidad de Antioquia, Medellín. Correspondencia: isabel.posada@udea.edu.co
}

2 Facultad de Ciencias Sociales, Universidad de Antioquia, Medellín. 


\begin{abstract}
Objectives: To analyze the perception of the university community of the context and risk factors associated with psychoactive substance abuse. Material and Methods: A historical and hermeneutic approach with qualitative design, and grounded theory as research methodology. Data collection was carried out using semi-structured interviews. A theoretical, convenience sampling was carried out. 18 interviews with the university community and professionals of the Students Welfare Office conducted. Analysis was carried out using coding, categorization, and interpretation. Results: The University is recognized as an institution that offers high quality education and research where individuals develop their views of life, the city, and the university. It is also a place where psychoactive substance abuse is facilitated given its environment, the ideas and lifestyles of its people, the concept of being a university student, and the existence of drug sales. Conclusion: Substance abuse is therefore a phenomenon that should be approached first locally, and then at national and international levels.
\end{abstract}

Key Words: Psychoactive Substances, Risk Factors, University Students.

Resumo

Objetivos: Analisar a percepção da comunidade universitária sobre o contexto e os fatores de risco associados ao consumo de sustâncias psicoativas. Materiais e métodos: enfoque histórico hermenêutico, desenho qualitativo, teoria fundamentada como método de pesquisa. Para a recoleção usou-se a entrevista semiestruturada. A amostragem foi teórica. Realizaram-se 18 entrevistas à comunidade universitária e profissionais de bem-estar universitário. Para a análise se utilizou a codificação, categorização e interpretação. Resultados: reconhece-se à universidade como uma instituição de qualidade em educação e investigação onde se assume uma posição ante a vida, a cidade e a universidade, e ao mesmo tempo como um cenário com elementos que facilitam o consumo: espaço físico, imaginários, estilos de vida, o que simboliza ser universitário e a venta de drogas. Conclusão: O consumo é, portanto um fenômeno que deve ser abordado desde uma perspectiva que transcenda do local ao nacional e internacional.

Palavras-chave: sustâncias psicoativas, fatores de risco, estudantes universitários.

\section{Introducción}

El uso de sustancias psicoactivas (SPA) ha estado presente en diferentes épocas y culturas. En la historia de la humanidad se encuentran asociadas a ritos, festejos y actividades cotidianas $\mathrm{y}$, en la actualidad, la diversidad de sus usos y contextos las presentan como un fenómeno de carácter global que afecta la salud pública. Ello sugiere la necesidad de contextualizar el fenómeno siguiendo la lógica internacional, nacional y local, hasta llegar específicamente a los campus universitarios, identificados como uno de los espacios de alto consumo.

En América Latina y el Caribe se destaca el primer estudio sobre el uso de drogas en población escolar secundaria, realizado en nueve países ${ }^{1}$ durante el año 2006, en el cual se

\footnotetext{
1 Argentina, Bolivia, Brasil, Colombia, Chile, Ecuador, Paraguay, Perú y Uruguay
} 
encontró que el alcohol en Colombia y Uruguay fue la droga lícita de mayor consumo; y la droga ilícita más consumida fue la marihuana en Chile. Los estudiantes manifestaron que el consumo de esta sustancia no era riesgoso (1). Aún con estos resultados, en esta investigación se evidencia un discurso con relación al consumo que se focaliza en la marihuana por los imaginarios y significados atribuidos al ser estudiante universitario.

En el Estudio epidemiológico andino sobre consumo de drogas sintéticas en la población universitaria. Informe Colombia (2009), se destaca que los estudiantes colombianos, en su mayoría, han consumido SPA alguna vez en la vida, principalmente la marihuana; los hombres son los mayores consumidores y el grupo de edad que más consume es el de 18 a 24 años (2). Con lo anterior, se puede afirmar que los universitarios de Colombia mantienen los índices más altos de consumo, si se comparan con los estudiantes de países como Bolivia, Ecuador y Perú. Además Colombia, país afectado por la producción, el tráfico y el consumo, presenta incrementos en estas problemáticas.

En la política nacional para la reducción del consumo de SPA y su impacto se plantea que "el consumo de SPA es un comportamiento que, cuando ocurre, dinamiza una serie de riesgos que al materializarse suelen provocar consecuencias adversas para el sujeto mismo, su familia y su comunidad" (3). La Gobernación de Antioquia, con el fin de conocer la realidad del consumo de drogas en el departamento, realizó, en asocio con Carisma, el estudio Prevalencia del consumo de sustancias psicoactivas y factores asociados mostrando que, en comparación con otras regiones, el Valle de Aburrá mantiene los más altos indicadores de consumo de SPA (4). El consumo en la comunidad académica de la Universidad de Antioquia (U de A) no se distancia de tal realidad, como tampoco de las lógicas internacionales, departamentales y locales, pues ella se configura como reflejo de la sociedad (5).

Situaciones de violencia, inseguridad y pobreza que se viven en la ciudad, se presentan en la universidad como variables causales y factores de riesgo asociados al consumo de SPA y que hacen más complejo su abordaje. Entendiendo los factores de riesgo como "cualquier característica o circunstancia detectable de una persona o un grupo de personas que se sabe asociada con un aumento de la probabilidad de padecer, desarrollar o estar especialmente expuesta a un proceso mórbido" (6). Estos factores de riesgo también se evidencian como prevalencia para el uso de drogas en un estudio realizado en 2008, en cuatro facultades de salud de la U de A (7).

Además, estudios de corte cuantitativo que en la actualidad evidencian altos índices de consumo de SPA en el país y, particularmente, entre la población universitaria, demandan ser complementados con la revisión de investigaciones cualitativas que, a pesar de ser pocas, profundizan en la mirada al fenómeno, los significados y percepciones asociadas a las SPA. Dos estudios cualitativos liderados por docentes y empleados de la universidad son de gran importancia y complementan la información sobre el consumo de SPA en la U de A.

En 2009, la Red para la Investigación, la Formación y la Prevención del Consumo de Sustancias Psicoactivas en el Escenario Universitario (Red Unir) realizó la investigación $\mathrm{Del}$ viaje en $U$, y entre sus resultados evidencian la representatividad de la marihuana en la vivencia universitaria, el posicionamiento de la heroína y el uso continuo de tranquilizantes y antidepresivos, todo asociado a un mercado de bajos precios que facilita el acceso (8). Por otro lado, se encuentra la investigación Representaciones sociales del consumo de "drogas" $y$ de 
las intervenciones respectivas en un contexto local: la Universidad de Antioquia, la cual ofrece claves interpretativas para la acción, relacionadas con creencias y prácticas en el consumo de drogas y sus intervenciones (9). Ambos estudios aportan información que contribuye a la comprensión de las representaciones e imaginarios que se asocian a las prácticas del consumo de los universitarios; sin embargo, Kumate plantea que, hasta la fecha, las investigaciones no han identificado todas las causas de las adicciones, factores y combinaciones más peligrosas, como tampoco se ha podido precisar sobre cuáles son más susceptibles de modificación; lo claro es que la etiología es compleja, multifactorial y que la predisposición al uso o abuso se relaciona directamente con el número de factores de riesgo a los cuales está expuesto el sujeto (10).

La Dirección de Bienestar Universitario a través del servicio de Prevención de las adicciones es el ente encargado de atender el consumo de SPA en la U de A y lo hace por medio de estrategias de formación, comunicación, investigación y orientación. Sin embargo, se observa baja acogida de estas y poco conocimiento sobre la percepción de los factores de riesgo por parte de la comunidad universitaria.

Teniendo en cuenta lo que dicen, piensan y significan las personas de la comunidad universitaria que a diario viven la universidad y su espacio físico e ideológico, este estudio pretendió analizar su percepción sobre el contexto y los factores de riesgo asociados al consumo de sustancias psicoactivas en la U de A, además aportará información para fortalecer los programas de prevención y promoción de estilos de vida saludables y el trabajo interdisciplinario entre la Comisión Interamericana para el Control del Abuso de Drogas (CICAD), la Organización de Estados Americanos (OEA) y la U de A.

\section{Materiales y métodos}

Se partió de un diseño cualitativo con el enfoque histórico hermenéutico. Se utilizaron herramientas de recolección y análisis propios de diferentes diseños cualitativos, como la entrevista semiestructurada y las técnicas de la teoría fundamentada, tales como la codificación, categorización y teorización. El análisis se realizó en tres momentos: descriptivo, analítico e interpretativo. Se utilizó un muestreo teórico, el cual ocurrió de manera secuencial a la recolección de los datos; se convocó a estudiantes de diversas facultades y en distintos momentos de su formación, así como personas de la comunidad académica, y se usó también la técnica de bola de nieve (11).

Los criterios de inclusión de los participantes fueron: a) ser estudiante de alguna facultad de la U de A; b) ser empleado docente de la U de A; c) ser empleado no docente vinculado con la U de A; d) ser empleado de Bienestar Universitario. La muestra estuvo conformada por 18 personas de las facultades de Ciencias Sociales y Humanas, Artes, Filosofía, Enfermería, Salud Pública, Ciencias Exactas, Ingeniería y Ciencias Agrarias. Lo anterior permitió hablar de una representatividad cualitativa en el estudio.

La técnica para la recolección fue la entrevista, en la cual se utilizó una guía semiestructurada con preguntas que giraban en torno a ejes temáticos relacionados con los objetivos del estudio: la situación del consumo de drogas en los contextos internacional, nacional y local, la percepción sobre los factores de riesgo asociados al consumo, la situación y respuesta ante el consumo en los universitarios.

El estudio se desarrolló en tres etapas; cada etapa contó con la participación como mínimo de un estudiante, un empleado docente y no docente y un profesional de Bienestar Universitario. Los investigadores se apoyaron en 
el programa Excel para la organización de los códigos y las categorías.

Primera etapa: se crearon dos guiones de entrevistas, uno para los estudiantes, docentes y empleados no docentes de la universidad y otro para los empleados de Bienestar. El procedimiento de transcripción, codificación abierta y análisis, permitió la emergencia de 19 categorías descriptivas que correspondían a la relación entre los contenidos o ejes temáticos.

Segunda etapa: se reformularon los primeros dos guiones de entrevista, diseñándose uno nuevo y único para toda la comunidad. Por medio de la codificación axial se cumplieron los propósitos planteados, la profundización de las categorías descriptivas y la relación entre las mismas. Con este proceso de análisis, emergieron cinco categorías analíticas, apoyadas en la elaboración de matrices, que permitieron identificar fenómenos centrales y sus contextos, causas, consecuencias y formas de presentación.

Tercera etapa: se reformuló el tercer guión. Se realizó la codificación selectiva con el fin de complementar, integrar y refinar las categorías analíticas de tal forma que se pudieran analizar y definir hasta llegar a cinco categorías interpretativas, las cuales permitieron organizar la información de acuerdo a los datos aportados por los informantes durante todo el proceso investigativo.

\section{Consideraciones éticas del estudio}

Para el desarrollo de esta investigación se tuvieron en cuenta los lineamientos de la Resolución 8430 de 1993 del Ministerio de Salud de Colombia sobre la investigación con seres humanos, con el fin de garantizar la integridad física y mental de los participantes (12). Cada persona entrevistada firmó un consentimiento informado, el cual contenía los objetivos y alcances de la investigación; los beneficios y la importancia de su participación. Así mismo, se tuvieron en cuenta los criterios establecidos en la Declaración de Helsinky de la Asociación Médica Mundial de 2008, donde se insta a los investigadores a garantizar la protección de la vida, la salud, la dignidad, la integridad, el derecho a la libre determinación, la privacidad y la confidencialidad de la información personal de los sujetos de la investigación (13).

\section{Resultados}

La categoría central que emergió fue nombrada como 'la U de A: un lugar para el saber y un escenario para el consumo'. Desde diferentes posiciones y experiencias enmarcadas en el vivir universitario, esta categoría da a conocer la percepción de la comunidad académica sobre el campus universitario como contexto para el consumo de SPA y los factores de riesgo asociados al mismo. Se abordan también diferentes temas que nutren e ilustran el contenido de la misma, siendo algunos de ellos causas para que se presente el fenómeno y otras consecuencias; a su vez, se plantean algunas actitudes que han de asumir los actores para disminuir los riesgos asociados al consumo, cuyo concepto no diferencia entre consumo problemático o uso en la gran mayoría de los entrevistados.

\section{La Universidad de Antioquia: un reflejo de la sociedad}

Las prácticas y problemáticas asociadas al consumo de SPA que se viven al interior de la $\mathrm{U}$ de A reflejan lo que sucede en la ciudad y a nivel nacional e internacional, pues esta no se puede considerar un ente aislado de la sociedad en la cual se halla inmersa. Al abordar el consumo de SPA, se deben particularizar realidades y estudiar los contextos en que se llevan a cabo estas prácticas. Así mismo, hay quienes insisten en la necesidad de intervenciones que se ajusten a las dinámicas propias de la institución. De esta manera, fue expresado por las personas de la 
comunidad universitaria: "de ahí vuelvo e insisto la necesidad de mirar el contexto, porque la universidad es el reflejo de lo que pasa en la sociedad y no solo en este tema, en el tema de corrupción y en el tema de problemáticas sociales" (E16D-83C84). ${ }^{2}$

Si bien el consumo de drogas es un problema de la sociedad, en el espacio universitario también se reflejan y legitiman prácticas asociadas al consumo de sustancias legales e ilegales. Los espacios de consumo al interior de la universidad convergen con los espacios de interacción intelectual y académica (9). Expresado de otra forma, la universidad se convierte para la sociedad en un lugar para el saber y un escenario para el consumo.

La universidad un lugar para el saber La U de A es la institución de educación superior más grande e importante del departamento de Antioquia, siendo además catalogada como la universidad estatal más antigua de Colombia y una de las mejores universidades del país, de acuerdo a la acreditación otorgada por el Consejo Nacional de Acreditación (CNA) de Colombia en septiembre de 2003, además de ser la segunda universidad del país en actividad investigativa, después de la Universidad Nacional de Colombia, según el Ranking Iberoamericano (SIR) 2014 sobre producción científica 20082012 (14).

De acuerdo al plan de desarrollo 2006-2016 de la universidad, su misión ha estado enmarcada en la formación integral del talento humano, con criterios de excelencia, la generación y difusión del conocimiento en los diversos campos del saber, y la preservación y revitalización del patrimonio cultural. Vista así, la universidad sigue cumpliendo el objetivo de academia, en-

2 Los números que aparecen después de los testimonios son los códigos con los que se identifican las entrevistas. señanza e investigación de excelencia, características que han acompañado desde sus inicios a las universidades como instituciones de educación superior. En este sentido, desde el alma máter se cuestionan aquellas actividades que van en contravía del debate, el conocimiento y la investigación; muestra de ello es el siguiente fragmento de un empleado de Bienestar:

"En un foro acá acerca de si era un problema o no el consumo, se le preguntó al magistrado Carlos Gaviria que es el ponente de la despenalización de la dosis personal: ¿usted cree que la universidad es donde se debe consumir? No, la universidad no es el espacio para consumir, la universidad es el espacio para hacer debate, para aprender, para la ciencia" (E4B-80C81).

La sociedad ha construido unos imaginarios o representaciones sociales sobre la U de A que trascienden de lo académico a lo social y lo cultural. Representaciones que tienen que ver con los estilos de vida, ideales, formas de relacionarse, consumos y vivencias universitarias. Estas representaciones coexisten y a través de los años han aportado a la construcción de la identidad de la universidad: "en realidad sí, la universidad es reconocida en muchas partes por su nivel académico como también es reconocida como los tira piedras, los consumidores" (E12A-46C47).

Lo anterior hace eco sobre un imaginario en particular, el de los consumidores. Son ellos quienes a través del tiempo han construido un entramado social y cultural frente al consumo de sustancias psicoactivas dentro del ambiente universitario. Ello implica realizar una visión retrospectiva a las formas en que sus actores construyeron el tejido social, que durante años, como prácticas habituales en el escenario universitario, han desarrollado el imaginario colectivo de aquello que es propio de sus estudiantes 
y que, en esa medida, se consolida como el referente cultural de la misma.

Existen posturas que adjudican no solo un ejercicio de construcción de conocimiento al espacio universitario, sino también que lo perciben como un escenario que permite la interacción social y facilita el consumo de SPA.

\section{La universidad un escenario para el} consumo

La comunidad universitaria reconoce que existen ciertos elementos de y en la universidad que pueden llegar a facilitar los consumos de SPA tanto legales como ilegales, entre ellos:

El espacio físico: 'el aeropuerto' y su significado El espacio físico del campus universitario, especialmente la zona deportiva a la cual durante décadas se le ha adjudicado el nombre de 'aeropuerto' es el principal elemento que, facilita el consumo de SPA, según los entrevistados. Por medio de la interacción empezaron a habitarlo, este lugar fue adquiriendo diversos usos, creando alrededor un conjunto de representaciones socialmente elaboradas y compartidas; otorgándole diversos significados, especialmente el de lugar de socialización. Recreando parte de la historia e imaginarios de la universidad, un docente expresa: "Porque siempre pues a nivel histórico y cultural se ha propiciado y se ha dejado permanecer un espacio para eso... Ni siquiera es que en la universidad se dé, sino que dentro de la universidad hay un espacio para eso que le llamamos 'el aeropuerto' y que eso está en el imaginario del colectivo universitario" (E5D-26-27C28).

Libertad y autonomía: significados otorgados al 'ser universitario'

Como segundo elemento en torno al cual los entrevistados desarrollaron la idea de la universidad como un escenario que reafirma la práctica de consumo, es el significado otorgado al'ser uni- versitario'. Ingresar a la U de A representa para los estudiantes libertad y autonomía personal; es así como el cambio del colegio a un ambiente universitario fue percibido por los entrevistados como el paso de la norma escolar y familiar a la libertad y la ausencia de límites. Lo anterior se ejemplifica con la opinión de un estudiante: "entonces el pelado que sale de 11 a explorar la vida universitaria, que tuvo toda la acción correctiva del colegio, que se mamó de eso, viene aquí y se abre y se expande, y juy voy a vivir la vida!" (E3E-156C157).

El consumo de marihuana, una percepción más de las prácticas universitarias

Desde la percepción de estudiantes, empleados y docentes, consumidores o no, se reconoce el consumo de marihuana en la $\mathrm{U}$ de $\mathrm{A}$, como una experiencia más del ser universitario, motivada por la curiosidad, el deseo de sentir placer o alejarse momentáneamente de la realidad; para otros, el consumir psicoactivos es una elección que repercute en sus vidas, lo que sugiere trascender la mirada de una práctica ligada al ciclo vital y específicamente a las características de ser joven y entender el consumo como decisiones que los estudiantes toman durante su proceso de formación.

"Porque tras de vivir expediciones de conocer, de hacerme preguntas, de mirar resultados, me he dado cuenta que el consumir marihuana no me hace ser menos productivo, no me limita pues mentalmente, no me conduce a cosas que yo no quiera elegir" (E10E-0103C104). En esta frase de un estudiante de la universidad, se evidencia que el consumo de marihuana no es un problema para algunos miembros de la comunidad; en lugar de ello, se le considera una práctica incorporada a los estilos de vida de los universitarios, que no obstaculiza su desarrollo académico y en el que se identifican conductas de autonomía, tal vez por ello, el consumo de 
otras sustancias, como el alcohol, no cobra notoriedad en el discurso de los entrevistados.

Si bien los entrevistados perciben que algunas dinámicas del campus universitario favorecen el consumo de SPA, consideran que esta situación no es determinante ni se puede generalizar a todas las personas que pertenecen a la U de A:

"Como te decía ahora, sí influye porque de todas formas cuando uno ingresa a la universidad viene y cambia, conoce nueva gente y trata de integrarse a esa nueva forma de vida y a ese nuevo grupo social, en ese sentido sí, pero el hecho de que una persona llegue y que porque llegue a la Universidad de Antioquia entonces es que va a empezar a consumir, no necesariamente" (E13B-43-44C50).

El expendio de drogas, el verdadero problema El conocimiento y la tolerancia que la comunidad universitaria había establecido frente a las dinámicas de consumo hasta hace pocos años, ha presentado transformaciones que se expresan en la preocupación generalizada respecto a las nuevas prácticas direccionadas hacia el expendio de drogas dentro de la universidad, las cuales sí se perciben e identifican como amenazas para el desarrollo, autonomía, confianza y pluralidad de los que habitan este escenario de conocimiento. El siguiente fragmento da cuenta del cuarto y último elemento que facilita el consumo en la U de A: "¿Un problema para quién o para qué? Yo pensaría que se debe mirar qué tipo de consumo, de pronto el consumo como tal no es el problema, el problema es ya los grupos organizados y de mafia que se están moviendo para la distribución y el abuso de las drogas en la universidad" (E5D-44C45).

La preocupación de los entrevistados gira entorno a los grupos organizados que distribuyen drogas al interior de la universidad, quienes olvidan el bienestar del sujeto consumidor y lo convierten en objeto que sostiene sus intereses particulares, y lo identifican como elemento útil para dar continuidad a prácticas delictivas. La demanda de la comunidad universitaria es sustituir las acciones de control dirigidas hacia los consumidores por acciones de prevención del consumo y promoción de la salud, y enfocar las primeras hacia los grupos y organizaciones que trafican al interior del campus universitario.

El consumo de SPA: ¿limita la libertad o posibilita su expresión?

Las consecuencias generadas por el expendio y el consumo problemático de sustancias psicoactivas se constituyen en uno de los problemas más importantes que tiene que afrontar la salud pública tanto nacional como internacional, dadas las complejas configuraciones que presenta este fenómeno.

Aparecen consecuencias individuales sociales derivadas del consumo, lo que afecta el desarrollo económico, humano, la seguridad de niños, jóvenes y adultos de un país. Para la U de A representa deserción estudiantil, pérdida de años de vida saludable y productiva de jóvenes con altas probabilidades de proyección social, deterioro de la calidad de vida y atraso para el desarrollo. Pareciera ser que los universitarios consumen para generar el vínculo social y como expresión de su libertad, sin darse cuenta que al traspasar la barrera del uso al abuso, tanto la libertad como la posibilidad de vínculo empiezan a desvanecerse. Un entrevistado lo expresa así: "Claro que la universidad es un espacio de libertad, sí pero es que el problema con las SPA es que no son una posibilidad de libertad sino todo lo contrario, el consumo de SPA puede, con el tiempo, sobre todo cuando se vuelve dependencia, convertirse, precisamente, en la pérdida de la libertad" (E4B-82C83).

Es por ello, que pensar en drogas no solo implica hablar del sujeto que consume, del con- 
texto en el que lo hace, de los diversos factores de riesgo, de las causas y sus consecuencias, también implica concebir estrategias de prevención y regulación del consumo.

\section{La responsabilidad: un compromiso} de todos con la universidad

Respecto a la responsabilidad frente al tratamiento del consumo de drogas al interior de la universidad, los estudiantes, docentes y empleados expresaron dos líneas de pensamiento que, aunque juntas, entran en contradicción; por un lado, se pretende y exige a Bienestar Universitario resultados positivos en cuanto a la disminución de las tasas de consumo y de las consecuencias asociadas a los consumos problemáticos, pero también se expresa que la responsabilidad por el devenir del fenómeno al interior de la universidad, es de todos y no solo de la Dirección de Bienestar.

Es por ello que el fenómeno de las drogas como tema transversal en la vida de los colombianos y de la comunidad universitaria, debe ser abordado desde una perspectiva que trasciende lo local y que se extienda a las sociedades. La idea es que desde la academia y entre todos se diseñen estrategias más incluyentes y mejor proyectadas para prevenir el consumo y promover los estilos de vida saludables; manteniendo la lógica de la misión institucional que convoca no solo a la formación intelectual sino a la construcción de individuos críticos y conscientes de las causas y consecuencias que a corto, mediano y largo plazo puede ocasionar la situación del consumo.

\section{Discusión}

Los puntos objeto de discusión en este artículo giran en torno a los siguientes apartados:

La universidad un reflejo de la sociedad El consumo de SPA dentro de la U de A, no se distancia de las lógicas internacionales y del diagnóstico que diferentes organizaciones sociales han adelantado en el país y en la misma ciudad de Medellín. El estudiante que ingresa a la $\mathrm{U}$ de $\mathrm{A}$ es un ciudadano y en la ciudad como tal se evidencian problemáticas, prácticas, estilos que acompañan a sus habitantes, de tal manera que lo que el individuo vive y hace en sus barrios probablemente será lo que se refleje en la universidad (5).

El consumo de drogas es un problema de la sociedad en general y no sólo de la U de A y esta, como reflejo de la sociedad, termina creando unos espacios que si bien no son legales sí se van legitimando y asumiendo como propios para el consumo (9).

Representaciones e imaginarios sobre la universidad

Los espacios físicos de la universidad adquieren significados diversos a través de la interacción social, algunas veces facilitando o mediando el consumo de SPA. De esta manera, el entorno urbano supera la dimensión física para adoptar también una dimensión simbólica y social (15).

Los resultados de esta investigación coinciden con lo encontrado por la Red Unir (8) que plantea que en el 'aeropuerto' se pueden configurar experiencias significativas de la vivencia universitaria, encuentros con los amigos, romances, 'despegues y aterrizajes', evoca la idea de un lugar abierto, amplio, donde se exprese y represente el hecho de ser universitario, lugar de expresión de la libertad. Las personas se apropian del espacio, transformándolo física y simbólicamente, al mismo tiempo que incorporan a sus estilos de vida determinadas cogniciones, afectos o actitudes relacionadas con el espacio que resultan parte fundamental de su identidad como individuos (15).

Regulación del consumo

Frente a la regulación del consumo de SPA, Olaya sugiere tres formas para llevar a cabo esta 
labor: la regulación normativa, la social y la autorregulación. La primera de ellas se refiere a las normas y leyes creadas por el Estado, la segunda implica, además de la responsabilidad individual, la adquisición de una conciencia colectiva y una responsabilidad con el otro, y la última es comprendida como un proceso en el que se percibe al individuo como un agente activo que a través de la toma de decisiones puede construir su destino $(16,17)$. En este mismo sentido, organizaciones internacionales, como la OEA, han presentado estrategias para hacerle frente a los problemas ocasionados por el consumo de SPA, estrategias que se enfocan en la prevención de riesgos y la regulación del consumo, a partir del reconocimiento del abuso y la adicción como problemas de salud pública, renunciando a posiciones como su erradicación o su criminalización como afrontamiento de la situación $(18,19,20)$.

Responsabilidad en la prevención del consumo y promoción de la salud La Red Unir plantea que, para la prevención del consumo de drogas y la promoción de la salud, es fundamental la integración de diferentes actores entre los cuales se encuentra Bienestar Universitario; sin embargo, las acciones deben involucrar a los gobiernos departamentales y municipales, y organizaciones comunitarias (8).

Por otro lado, el consumo demanda un abordaje en dos frentes: la prevención y el tratamiento. La primera, requiere programas bien diseñados que trasciendan las administraciones universitarias y, para ello, es necesario un compromiso de largo aliento y una participación de todos los estamentos; además, se requiere coherencia: el campus universitario debe ser territorio libre de consumo de todo tipo de sustancias adictivas, como alcohol y cigarrillo, $\mathrm{y}$ las autoridades universitarias deben dar ejemplo de ello. A los adictos se les debe ofre- cer tratamiento rehabilitador integral por parte de equipos profesionales expertos (21).

\section{Conclusiones}

Las intervenciones en SPA en la universidad deben responder a las necesidades de la comunidad, sin desconocer el aporte de las políticas nacionales e internacionales.

Percibir los riesgos para consumir SPA en la comunidad universitaria no es suficiente para evitar el consumo, se deben revisar e intervenir las subjetividades de quienes tienen este tipo de práctica.

La universidad puede ser un contexto facilitador pero no determinante del consumo de SPA, por su espacio físico, sus imaginarios, lo que simbolizan sus prácticas y el expendio de drogas dentro del campus.

Existe una serie de factores de riesgo que, sumados entre sí, pueden incrementar la probabilidad de consumir SPA; sin embargo, se deben tener en cuenta características personales que, a su vez, se convierten en factores protectores para esta práctica.

Si bien el fenómeno investigado se contextualizó en la Universidad de Antioquia, no se puede desconocer que este es de carácter global y se debe abordar desde una perspectiva que trascienda de lo local a lo nacional e internacional.

Prevenir el consumo y promocionar los estilos de vida saludables en la U de A no es solo responsabilidad de la Dirección de Bienestar sino de toda la comunidad universitaria, en pro de la misión de construir individuos críticos.

\section{Agradecimientos}

A la Estrategia de Sostenibilidad CODI 20132014 de la Universidad de Antioquia.

Este proyecto fue financiado por la CICAD y se desarrolló en el convenio que existe entre la Facultad Nacional de Salud Pública (FNSP) 
desde el año 2007 con la Comisión Interamericana para el Control del Abuso de Drogas
(CICAD) de la Organización de Estados Americanos (OEA).

\section{Bibliografía}

1. Wright MG, Chisman A, Carletto A, Aver A, Restrepo MV, Rojas X, et al. . El conocimiento y las actividades de los docentes en salud pública en el fenómeno de las drogas y salud internacional en América Latina. Washington: Cicad, OEA, OPS; 2009.

2. Ministerio del Interior y de Justicia, Dirección Nacional de Estupefacientes. Estudio epidemiológico andino sobre consumo de drogas sintéticas en la población universitaria, Informe Colombia [internet]. 2009 [citado 2011 nov 11]. Disponible en http://www.comunidadandina.org/Upload/201161193158Estudio_drogas.pdf

3. Ministerio de la Protección Social, Dirección General de Salud Pública. Política nacional para la reducción del consumo de sustancias psicoactivas y su impacto. Resumen ejecutivo [internet]. 2007 [citado 2011 ago 21]. Disponible en: http://www.descentralizadrogas.gov.co/portals/0/Politica \%20nacional\%20SPA.pdf

4. Gobernación de Antioquia, Carisma. Prevalencia de consumo de sustancias psicoactivas y factores asociados. Resultados de la investigación en jóvenes escolarizados del Departamento de Antioquia [internet]. 2003 [citado 2011 jul 3]. Disponible en: http://www.odc.gov.co/Portals/1/publicaciones/pdf/consumo/ estudios/locales/CO031052003-prevalencia-consumo-de-sustancias-psicoactivas-factores-asociados.pdf

5. Osorio P. Alas rotas. ¡Qué pasa UdeA!, especial periodístico [internet]. 2010 [citado 2011 sep 21]. Disponible en: http://periodistasudea.com/quepasaudea/2010/alas-rotas/

6. Díaz MP. El riesgo en salud: entre la visión del lego y del experto. Una perspectiva sociocultural. Bogotá: Universidad Nacional de Colombia; 2002.

7. Montoya EM, Cunningham J, Brands B, Strike C, Wright MG. Consumo percibido y uso de drogas lícitas e ilícitas en estudiantes universitarios en la ciudad de Medellín Colombia. Rev Latino-Am Enfermagem. 2009; 17(Esp):886-92.

8. Red para la Investigación, la Formación y la Prevención del Consumo de Sustancias Psicoactivas en el Ámbito Universitario. Del viaje en U. La vivencia universitaria y el consumo de sustancias psicoactivas. Medellín: Red Unir; 2009.

9. Henao S. Representaciones sociales del consumo de "drogas" y de las intervenciones respectivas en un contexto local: la Universidad de Antioquia en Medellín, Colombia. [tesis doctoral]. [Granada]: Universidad de Granada; 2010. 397 p.

10. Kumate J. Percepción de riesgo y consumo de drogas en jóvenes mexicanos [internet]. 2002 [citado 2011 ago 23]. Disponible en: http://www.salud.gob.mx/unidades/cdi/documentos/jn2602pers.pdf

11. Strauss A, Corbin J. Bases de la investigación cualitativa. Técnicas y procedimientos para desarrollar una teoría fundamentada. Medellín: Editorial Universidad de Antioquia; 2002.

12. Colombia, Ministerio de Salud. Resolución 8430 de 1993, Por la cual se establecen las normas científicas, técnicas y administrativas para la investigación en salud (1993 oct 4).

13. Asociación Médica Mundial. Declaración de Helsinki de la Asociación Médica Mundial. Hong Kong: $41^{a}$ Asamblea Médica Mundial; 1989.

14. Scimago Lab. SIR Iber 2014. Rank: Output 2008-2012. [internet] s. f. [citado 2014 jun 11]. Disponible en: http://www.scimagoir.com/pdf/iber/SIR\%20Iber\%202014\%20HE.pdf

15. Valera S, Pol E. El concepto de identidad social urbana: una aproximación entre la psicología social y la psicología ambiental. Anu Psicol. 1964; 62:5-24. 
16. Olaya A. Antecedentes históricos culturales del consumo de alcohol. Documento presentado en: Simposio Consumo Moderado de Alcohol. 2011 abr 15; Medellín.

17. García JA, Días P. Análisis relacional entre los factores de protección, resiliencia, autorregulación y consumo de drogas. Salud y drogas, 2007; 7(2):309-32.

18. Organización de Estados Americanos. Comisión Interamericana para el Control del Abuso de Drogas Cicad. Estrategia hemisférica sobre drogas [internet]. 2010 [citado 2014 jun 11]. Disponible en: http:// www.cicad.oas.org/apps/Document.aspx?Id=954

19. Organización de Estados Americanos. El problema de las drogas en las Américas [internet]. 2013 [citado 2014 jun 11]. Disponible en: http://www.oas.org/documents/spa/press/Introduccion_e_Informe_Analitico.pdf

20. Organización de Estados Americanos. Escenarios para el problema de las drogas en las Américas. 20132025 [internet]. 2012 [citado 2014 jun 11]. Disponible en: http://www.oas.org/documents/spa/press/ Informe_de_Escenarios.pdf

21. Cano VH, Patiño CD. Representaciones sociales sobre el consumo de sustancias psicoactivas por parte de jóvenes consumidores de la ciudad de Medellín entre 2006-2008 [internet]. 2009 [citado NC Falta esta información]. Disponible en: http://bibliotecadigital.usbcali.edu.co:8080/jspui/bitstream/10819/307/1/ Representaciones_Sociales_Consumo_Cano_2009.pdf 